\title{
OBSERVATIONS OF AGN WITH THE EXTREME ULTRAVIOLET EXPLORER
}

\author{
HERMAN L. MARSHALL \\ Eureka Scientific, Inc. \\ 2452 Delmer St, Suite 100 \\ Oakland, CA 94602 \\ and \\ Massachusetts Institute of Technology \\ 70 Vasser St., 37-667a \\ Cambridge, MA 02139
}

\begin{abstract}
The first results from surveys performed in the extreme ultraviolet (EUV) will be described in the context of studies of active galaxies and BL Lac objects. About a dozen extragalactic sources are known so far to emit sufficient EUV radiation that they are detectable even through the Galactic interstellar medium. These results are interpreted in the context of a model of EUV or soft X-ray excesses in the case of AGN. In the case of BL Lac objects, the detections indicate that the steep soft X-ray power law spectra continue into the EUV and that there is little intrinsic gas. Finally, there now exists EUV spectra from the Extreme Ultraviolet Explorer for one BL Lac, PKS 2155-304 and two AGN: Mk 478 and NGC 5548. The spectra show no significant spectral features; for AGN, it indicates that optically thin and emission line models may have a difficult time explaining the EUV and soft X-ray bumps.
\end{abstract}

Key words: AGN, BL Lac Objects

\section{Introduction}

When I first started on the Extreme Ultraviolet Explorer (EUVE) project, it was painfully aware to all extragalactic astronomers that it would be nearly impossible to observe galaxies or their active nuclei (AGN) in the extreme ultraviolet (EUV), sometimes defined as the range of wavelengths between the boron $\mathrm{K}$ edge at $68 \AA$ and the Lyman $\alpha$ edge at $1216 \AA$. The basic reason was that the interstellar medium (ISM) was known to present a large optical depth longward of $100 \AA$ : for a typical low value of $N_{H} \sim 2 \times 10^{20} \mathrm{~cm}^{-2}$, the optical depth out of the Galaxy would be about 7.

My personal view on this subject was turned around by two papers. First, Lockman, Jahoda, and McCammon (1986) showed that there are regions where the $N_{H}$ out of the Galaxy is very small, less than $10^{20} \mathrm{~cm}^{-2}$ in some cases. Second, Wilkes and Elvis (1987) showed that AGN often appear to have soft X-ray excesses that compensate for ISM absorption. With these two observations and some quantitative representations about them, I made the first predictions at the EUV conference at Berkeley in January, 1989 (Marshall, 1991). Surprisingly, I found that of order 100 AGN would be found in the EUVE and ROSAT Wide Field Camera (WFC) all-sky surveys. Only the shortest wavelength bandpasses, with the Lexan/B filter

105

T. J.-L. Courvoisier and A. Blecha: Multi-Wavelength Continuum Emission of AGN, 105-110.

(C) 1994 IAU. Printed in the Netherlands. 


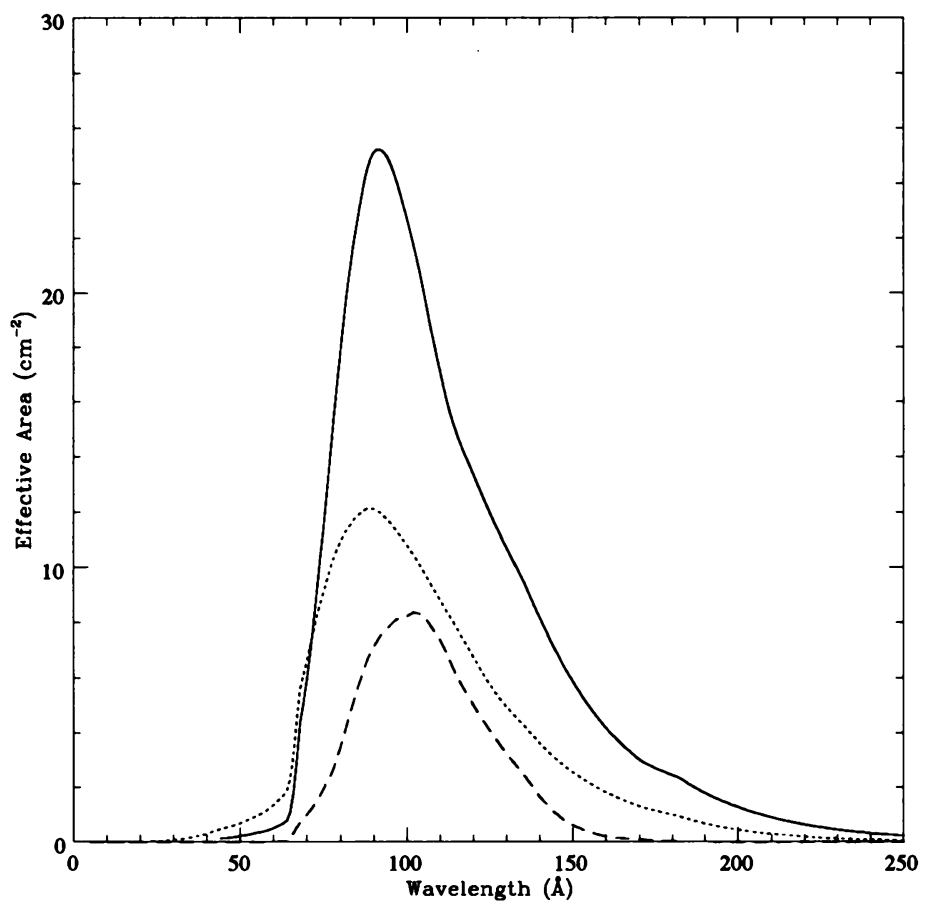

Fig. 1. Effective area functions for three short wavelength telescopes. Solid line: EUVE Deep Survey Lexan/B band. Dotted line: EUVE Scanner Lexan/B band. Dashed line: ROSAT WFC Lexan/B band. Note that the EUVE survey telescope has a much broader bandpass than the WFC.

(figure 1), are expected to allow detection of AGN because the ISM is too opaque longward of about $150 \AA$.

\section{EUV Survey Results}

The ROSAT WFC all-sky survey was completed in January, 1991. There were about 380 sources, of which 4 were identified as AGN and 3 were BL Lac objects (Pounds, et al., 1993). In the EUVE all-sky survey, completed in January, 1993 (although gaps were filled until July 1993), 11 AGN and 5 BL Lacs were detected (Marshall, Carone, and Fruscione, 1994). See Table I for the list of known sources and their count rates in these surveys. Note that the WFC results from Pounds, et al. are for the complete survey, so sources have to be bright enough to satisfy the criterion for sources independent of the possible identification. There are data in the table from Balucinska-Church and Gondhalekar (1992) as well as Marshall, Carone, and Fruscione, who select targets on the basis of optical or X-ray properties 
and then determine the possible flux from the target; this procedure picks up sources at fainter flux levels than the surveys.

TABLE I

AGN and BL Lacs Detected in EUV Surveys

\begin{tabular}{|c|c|c|c|}
\hline Name & $\begin{array}{c}\text { Type }^{a} \\
(\mathrm{Q}, \mathbf{A}, \mathbf{B})\end{array}$ & $\begin{array}{l}R_{W F C} \\
(\mathrm{cnt} / \mathrm{s})\end{array}$ & $\begin{array}{l}R_{E U V E} \\
\text { (cnt/s) }\end{array}$ \\
\hline Q0239-591 & $\bar{Q}$ & & 0.019 \\
\hline H0425-573 & $\mathbf{A}$ & & 0.050 \\
\hline BL1011+496 & B & & 0.021 \\
\hline $\mathrm{X} 12325^{b}$ & $\mathbf{A}$ & 0.021 & 0.017 \\
\hline $\operatorname{Mk} 421^{b, c}$ & B & 0.069 & 0.059 \\
\hline PG1116+215 & $\mathbf{Q}$ & & 0.031 \\
\hline NGC 4051 & $\mathbf{A}$ & $<0.010^{e}$ & 0.016 \\
\hline $3 \mathrm{C} 273$ & $\mathbf{Q}$ & $0.008^{e}$ & 0.036 \\
\hline Mk $279^{c}$ & $\mathbf{A}$ & & 0.043 \\
\hline NGC 5548 & $\mathbf{A}$ & $0.010^{e}$ & $0.027^{d}$ \\
\hline IC $3599^{b}$ & $\mathbf{A}$ & 0.013 & $<0.005$ \\
\hline Mk $478^{b}$ & $\mathbf{A}$ & 0.052 & $0.048^{d}$ \\
\hline $\mathrm{H} 1426+427^{b}$ & B & 0.013 & 0.029 \\
\hline Mk $501^{c}$ & B & & 0.032 \\
\hline PKS2155-304 ${ }^{b, c}$ & B & 0.166 & 0.249 \\
\hline NGC 7213 & $\mathbf{A}$ & $<0.008^{e}$ & 0.033 \\
\hline $\mathrm{RE}_{2248-511^{b}}$ & $\mathbf{A}$ & 0.018 & 0.020 \\
\hline
\end{tabular}

${ }^{a} \mathrm{~A}=\mathrm{AGN}, \mathrm{Q}=$ quasar, $\mathrm{B}=\mathrm{BL}$ Lac object

${ }^{b}$ Source is in WFC bright source list (Pounds, et al., 1993).

${ }^{c}$ Source is in EUVE bright source list and satisfies the $6 \sigma$ selection criterion (Malina et al., 1994).

${ }^{d}$ Source was observed during EUVE survey gap fill-in.

${ }^{e}$ Source data are taken from Balucinska-Church and Gondhalekar (1992).

There are a few features of the list to note. First, the BL Lacs that are bright in the hard X-ray band show up well in the EUVE bandpass. This is probably due to the steep spectra of BL Lacs and that they probably have very little neutral gas intrinsic to the host galaxy. Similarly, BL Lacs make up a relatively large fraction of the population, $30 \%$, although they comprise less than $15 \%$ of the population at hard X-rays (Remillard, 1991).

Second, there are not as many AGN as expected before launch. There are two reasons for this, which are different for the two surveys. For the WFC, the Lexan/B effective area was about a factor of three lower than the pre-launch estimates. Furthermore, the average sensitivity was expected to be about 0.01 count/s and it turned out closer to about 0.02 count/s. Taking the sky coverage function into account (Pounds, et al., 1993), I repeated the prediction using the method from Marhshall (1991) to obtain 4.5 sources expected in the WFC survey. They observed 4 , so this model apparently works. The model is relatively simple: a power law 
with $f_{n u} \propto \nu^{-\alpha}$ and $\alpha=1$ is augmented with a thermal component with $T \sim$ $8 \times 10^{5} \mathrm{~K}$ that would match UV spectra. The Galactic absorption was handled with a simple distribution function fitted to low $N_{H}$ data (from F. J. Lockman, private communication; the data are also given in Marshall 1991).

In the case of the EUVE survey, however, the prediction is 9.7, given the actual sky coverage function in the bright source list (Malina et al., 1994). There is significant incompleteness, however, so the final value is expected to be much closer to the observed number: 2 AGN brighter than the $6 \sigma$ threshold required for the EUVE bright source list. Again, the sensitivity of the survey was significantly worse than expectations, decreasing the predicted number of AGN.

\section{Results from Observations of Individual Sources}

\subsection{PKS 2155-304}

This bright BL Lac object was observed with the EUVE Deep Survey/Spectrometer during the EUVE in-orbit checkout phase in early July, 1992 (before the survey started). It was known to be the brightest extragalactic source in the WFC survey and was chosen because there would be very little long wavelength flux so that the second order response of the EUVE spectrometer could be verified. The source was not strongly variable during the EUVE observations and simultaneous optical data indicated that the overall spectral energy distribution decreased by about a factor of two between in the two years between the EUVE and WFC observations (Marshall, Carone, and Fruscione, 1993). Furthermore, with an accurate value of $N_{H}, 1.36 \times 10^{20} \mathrm{~cm}^{-2}$ (Lockman and Savage, 1993), they showed that the count rates of PKS 2155-304 were consistent with a spectral model with an ISM HeI/H ratio of 0.07 to 0.10 and $\alpha=1.66$, as observed in the soft X-ray band (Fink et al., 1992). Using nearly the same spectral index and $N_{H}$ values Fruscione et al. (1993) showed that additional neutral gas, $\sim 3 \times 10^{19} \mathrm{~cm}^{-2}$, would be required to fit the EUVE spectrometer data if one requires $\mathrm{HeI} / \mathrm{H}=0.07$ (which is favored by other EUVE observations).

\subsection{NGC 5548}

This AGN was detected by the WFC (Balucinska-Church and Gondhalekar, 1992) and was observed in March and May, 1993. The observations were coordinated with HST and ground-based optical observations so that correlations of the emission lines and the ionizing continuum could be obtained to look for lags. The source was extremely faint, however, so the planned exposure for the observation was 600,000 s, or a total observatory time of about 23 days.

A preliminary analysis has been presented at this conference (Kaastra et al., 1993). The source is so weak that the continuum is practically invisible. There is a hint of emission near $95 \AA$ but it is improbable that this flux would be detectable through the Galactic ISM, estimated at $N_{H}=2 \times 10^{20} \mathrm{~cm}^{-2}$, especially if there is no detection at $75 \AA$ or shorter. 


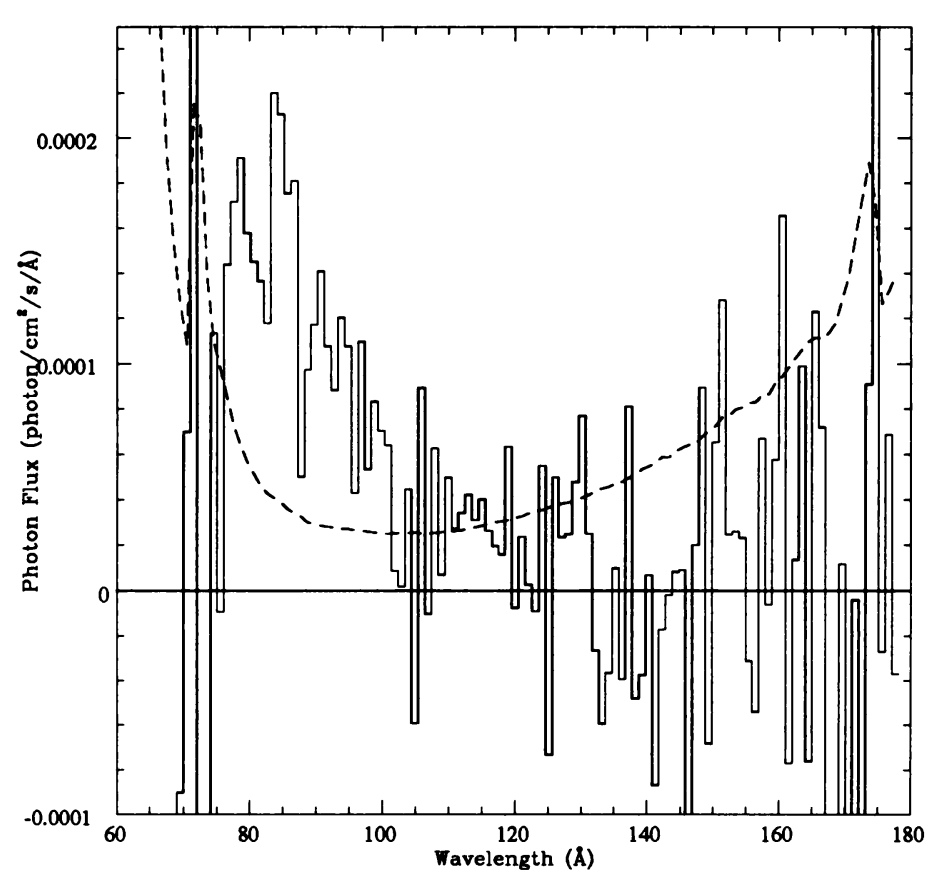

Fig. 2. EUVE spectrum of Mk 478. Solid line: Background subtracted photon flux in $1 \AA$ bins. Dashed line: Uncertainty in photon flux. There are no significant emission features at the resolution of the spectrometer, which is less than $1 \AA$. As expected, the flux cuts off at long wavelengths due to the opacity of the Galactic ISM. Note that the uncertainties are very high shortward of $75 \AA$, which is due to scattered geocoronal Lyman $\alpha$, and that the continuum is marginally detectable in the $100-120 \AA$ range. The spike at $71.5 \AA$ is due to poor background subtraction at the bright detector rim.

\section{3. $\mathrm{MK} 478$}

The AGN Mk $478(z=0.079)$ was observed with the EUVE spectrometer in early April, 1993. It was proposed because it was the brightest AGN in the WFC survey and the spectrum shows that it was well detected (figure 2).

There are no significant features in the spectrum, so the first impression (the spectrum is only a week old) is that the EUV excesses are not due to a collection of emission lines, a possibility that may account for some soft X-ray excesses (Turner et al., 1991). Furthermore, there are no narrow emission features that might accompany a thin thermal plasma, if the UV bump and soft X-ray excesses are to be fitted with a bremstrahllung model. Detailed analyses will place stringent limits on the amounts of gas that may be present with temperatures between $10^{5}$ and $10^{6.5} \mathrm{~K}$ (Marshall et al., 1994). 


\section{Summary}

In conclusion, current EUV instruments are capable of detecting AGN and BL Lac objects so that we have a new diagnostic. The spectroscopic data are especially exciting because we finally have sufficient resolution to test models of the soft X-ray excess.

\section{Acknowledgements}

I thank Antonella Fruscione for providing me the spectrum of PKS 2155-304 to present and Jelle Kaastra for two preliminary figures from the NGC 5548 analysis. This work has been supported by NASA: NAS5-32485 to Eureka Scientific, Inc., M.I.T. subcontract SV1-61010 under SAO contract NAS8-39073, and NAS5-30180 to U.C. Berkeley.

\section{References}

Balucinska-Church, M., and Gondhalekar, P. 1992, in X-ray Emission from Active Galactic Nuclei and the Cosmic $X$-ray Background, ed. W. Brinkmann, and J. Trümper, (Garching: MPE), 1992, 224.

Fink, H. H., Thomas, H.-C., Brinkmann, W., Okayashu, R., and Hartner, G. 1992, in X-ray Emission from Active Galactic Nuclei and the Cosmic X-ray Background, ed. W. Brinkmann and J. Trümper, (Garching: MPE), 1992, 202.

Fruscione, A., Bowyer, S., Konigl, A., Kahn, S. 1993, Ap. J. (Letters), submitted.

Kaastra, J., et al. 1993, these proceedings.

Lockman, F.J., Jahoda, K., and McCammon, D., 1986, Ap. J., 302, 432.

Lockman, F.J. and Savage, B. 1993 Ap. J. Suppl., submitted.

Malina, R.F., et al. 1994, Astron. J., accepted.

Marshall, H. L. 1991, in EUV Astronomy, R. F. Malina and C. S. Bowyer, eds., (New York: Pergammon), p. 228.

Marshall, H.L., Carone, T.E., and Fruscione, A. 1993, Ap. J. (Letters), 414, L53.

Marshall, H.L., Carone, T.E., and Fruscione, A. 1994, Ap. J., submitted.

Marshall, H.L., Carone, T.E., Shull, J.M., Malkan, M., Elvis, M., and Green, R.F. 1994, in preparation.

Pounds, K., et al. 1993, M.N.R.A.S., 260, 77.

Remillard, R. 1991, MC-LASS catalog, unpublished.

Turner, T.J., Weaver, K.A., Mushotzky, R.F., Holt, S.S., and Madejski, G.M. 1991 Ap. J., 381, 85.

Wilkes, B.J. and Elvis, M. 1987, Ap. J., 323, 243. 